\title{
GENERALIZED QUASILINEAR HYPERBOLIC EQUATIONS AND YOSIDA APPROXIMATIONS
}

\author{
JONG YEOUL PARK, IL HYO JUNG and YONG HAN KANG
}

(Received 27 January 2001; revised 31 October 2001)

Communicated by K. Wysocki

\section{Abstract}

We will show the existence, uniqueness and regularity of global solutions for the Cauchy problem for nonlinear evolution equations with the damping term

$$
u^{\prime \prime}(t)+M\left(\left|A^{1 / 2} u(t)\right|^{2}\right) A u(t)+\delta u^{\prime}(t)=f(t) \quad(\delta>0) .
$$

As an application of our results, we give the global solvability and regularity of the mixed problem with Dirichlet boundary conditions:

$$
u^{\prime \prime}(x, t)+(-1)^{k} M\left(\int_{\Omega}\left|\nabla^{k} u(x, t)\right|^{2} d x\right) \Delta^{k} u(x, t)+\delta u^{\prime}(x, t)=f(x, t) .
$$

2000 Mathematics subject classification: primary 35A05, 35L70.

Keywords and phrases: Yosida approximations, quasilinear hyperbolic equation, global solutions, regularity.

\section{Introduction}

We consider abstract Cauchy initial value problems in a Hilbert space $H$ for nonlinear evolution equations of the form

$$
\left\{\begin{array}{l}
u^{\prime \prime}(t)+M\left(\left|A^{1 / 2} u(t)\right|^{2}\right) A u(t)+\delta u^{\prime}(t)=f(t) \text { on }(0, \infty), \\
u(0)=u_{0}, \quad u^{\prime}(0)=u_{1},
\end{array}\right.
$$

where the operator $A$ and the function $M(\cdot)$, which satisfy convenient assumptions, are given, and $\delta>0$ is a constant.

This work was supported by the Brain Korea 21 Project, 1999.

(C) 2003 Australian Mathematical Society 1446-7887/03 \$A2.00+0.00 
The problem (CP) has attracted the attention of several researchers (see $[1-4,6-$ 10,12 ] and references therein) because of its intimate connection with mathematical physics. In fact, we consider the following nonlinear partial integro-differential equation

$$
u^{\prime \prime}(x, t)-\left(\alpha+\beta \int_{0}^{1}|D u(x, t)|^{2} d x\right) D^{2} u(x, t)+\delta u^{\prime}(x, t)=f(x, t),
$$

for $x \in(0,1)$ and $t>0$, subject to the boundary conditions

$$
u(0, t)=u(1, t)=0, \quad t>0,
$$

with initial conditions

$$
u(x, 0)=u_{0}(x), \quad u^{\prime}(x, 0)=u_{1}(x), \quad x \in(0,1),
$$

where $D=d / d x, D^{2}=d^{2} / d x^{2}$ and $\alpha, \beta, \delta$ are positive constants.

Then it is well known that the problem (1.1)-(1.3) describes the damped small amplitude vibrations of an elastic and stretched string (see [1,4]). Actually, the problem (1.1)-(1.3) can be written abstractly as the problem (CP) and hence solutions $u(t)$ to the problem (CP) can be regarded as solutions to the problem (1.1)-(1.3) by considering pointwise evaluation of solutions, $(u(t))(x)=u(x, t)$.

When $\delta=0$, Dickey [2] and Pohožaev [10] have shown the existence and uniqueness of local solutions to the problem (CP) with $A=-\Delta$ and $A^{k}=(-\Delta)^{k}$ by using a Galerkin procedure, respectively (see also [6]). In case of $A=-\Delta$ in $L^{2}\left(\mathbb{R}^{n}\right)$, Yamada [12] has shown the existence of global solutions to the problem (CP) with small data by using an iteration procedure. Later, Ikehata and Okazawa [4] have obtained global solutions to the problem (CP) by using the Yosida approximation method under compactness argument and the special small initial data. We note that in this paper, the Yosida approximation of $A$ plays a central role in deriving some a priori estimates of solutions to the problem (CP).

The purpose of this paper is to show the existence and uniqueness of global solutions to the problem (CP) under the presence of the damping term $\delta u^{\prime}(t)(\delta>0)$. We will study the regularity of solutions. Moreover, as a general result of Pohožaev [10], considering an operator $A^{k}$ instead of $A$ in (CP), we will also show the existence and the regularity of solutions. The proof of the solvability of the problem (CP) is carried out by the Yosida approximation method. Note that result of this paper is a relative generalization of $[1]$ and $[10,12]$ with $A=-\Delta$ in $L^{2}(\Omega)$ as a special operator. Furthermore our study may be extended to more general situations. For example, we can apply our methods to the generalized damped extensible beam equation of the form:

$$
u^{\prime \prime}(t)+A^{2} u(t)+M\left(\left|A^{1 / 2} u(t)\right|^{2}\right) A u(t)+\delta u^{\prime}(t)=f(t)
$$


The content of this paper is as follows. In Section 2, we give the abstract setting and main results. In Section 3, we mention some useful facts about Yosida approximation of a nonnegative selfadjoint operator. Section 4 and Section 5 are devoted to the proof of our main results and an application for our abstract results.

\section{Abstract setting and results}

Let $H$ be a real Hilbert space with an inner product $(\cdot, \cdot)$ and a norm $|\cdot|$. Suppose $A$ is a densely defined nonnegative selfadjoint linear operator in $H$. Then the powers of degree $\gamma>0$ of $A, A^{\gamma}$, may be computed via the elementary spectral calculus and are known to be nonnegative selfadjoint operators themselves. In fact, $A^{\gamma}$ is defined by

$$
A^{\gamma}=\int_{0}^{\infty} \lambda^{\gamma} d E(\lambda) \quad \text { with domain } D\left(A^{\gamma}\right),
$$

which means that $\left(A^{\gamma} u, v\right)=\int_{0}^{\infty} \lambda^{\gamma}(d E(\lambda) u, v)$ holds for all $u \in D\left(A^{\gamma}\right)$ and $v \in H$, where $\{E(\lambda): 0 \leq \lambda<\infty\}$ is the resolution of the identity associated with $A$ (for details, see [11]).

Note that in this case, the resolution of the identity $\{E(\lambda)\}_{\lambda \geq 0}$ is uniquely determined so that $A^{\gamma}$ can be represented as (2.1) and also it is well known that $D\left(A^{\gamma}\right)$ is a real Hilbert space with the graph norm $|v|_{D\left(A^{\gamma}\right)}^{2}=|v|^{2}+\left|A^{\gamma} v\right|$ and for $0 \leq \gamma_{1} \leq \gamma_{2}$,

$$
D\left(A^{\gamma_{2}}\right) \subset D\left(A^{\gamma_{1}}\right) \text {. }
$$

Now we shall consider nonlinear evolution equations of the form

$$
\begin{aligned}
& u^{\prime \prime}(t)+M\left(\left|A^{1 / 2} u(t)\right|^{2}\right) A u(t)+\delta u^{\prime}(t)=f(t) \text { in } H, \\
& u(t) \in D(A) \text { for any } t \in[0, \infty), \\
& u(0)=u_{0} \in D(A), \quad u^{\prime}(0)=u_{1} \in D\left(A^{1 / 2}\right) .
\end{aligned}
$$

Throughout what follows we will use the notation $V:=D(A)$ and $W_{l}:=D\left(A^{l / 2}\right)$, respectively. In particular, we let $W_{1}:=W$ and $W_{0}:=H$.

We assume the following about $A, M(t)$, and $f(t)$ :

(A.1) $A$ is a nonnegative selfadjoint operator and the injection from $W$ into $H$ is compact.

(A.2) $M(t)$ is a $C^{1}[0, \infty)$ class function satisfying $M(t) \geq m_{0}$ ( $m_{0}>0$ is a constant). (A.3) $f \in L^{1}(0, \infty ; W) \cap L^{\infty}(0, \infty ; H)$.

For the later use, we set

$$
\bar{M}(r)=\int_{0}^{r} M(s) d s, \quad E(t)=\frac{1}{2}\left[\left|u^{\prime}(t)\right|^{2}+\bar{M}\left(\left|A^{1 / 2} u(t)\right|^{2}\right)\right] .
$$


Note that $E(t)$ may be regarded as the total energy of the problem (2.3)-(2.5) at time $t$ and by assumption (A.2),

$$
\bar{M}(r) \geq m_{0} r \text { on }[0, \infty) .
$$

Definition 2.1. A function $u(t):[0, T) \rightarrow H$ is called a solution for the problem (2.3)-(2.5) on $[0, T)(T$ may be $\infty)$ if

(i) $u \in L^{\infty}(0, T ; V) \cap B C([0, T) ; W), u^{\prime} \in L^{\infty}(0, T ; W) \cap B C([0, T) ; H)$, $u^{\prime \prime} \in L^{\infty}(0, T ; H)$;

(ii) $u$ satisfies $(2.3)$ on $[0, T)$;

(iii) $u(0)=u_{0}$ and $u^{\prime}(0)=u_{1}$.

Here $B C([0, T) ; H)$ denotes the set of all $H$-valued bounded continuous functions on $[0, T)$.

We put $D(\gamma):=\left\{\left(u_{0}, u_{1}, f\right) ;\left|u_{0}\right|_{v} \leq \gamma,\left|u_{1}\right|_{W} \leq \gamma, \int_{0}^{\infty}|f(t)|_{W} d t \leq \gamma\right\}$. We now state the main result.

THEOREM 2.1. Let all assumptions (A.1)-(A.3) be satisfied and $\left(u_{0}, u_{1}, f\right) \in V \times$ $W \times L^{1}(0, \infty ; W)$. Then there exists $\gamma_{0}\left(\gamma_{0}>0\right)$ satisfying the following. If

$$
\left(u_{0}, u_{1}, f\right) \in D\left(\gamma_{0}\right),
$$

then there exists a unique solution $u(t)$ on $[0, \infty)$ to the problem $(2.3)-(2.5)$.

REMARK 2.1. (i) When $M(t) \geq \alpha+\beta t(\alpha>0, \beta>0)$ and $f(t) \equiv 0$, our result coincides with that of [1]. Thus this may be a generalization of the result in [1].

(ii) As a special condition on $D(\gamma)$, Ikehata and Okazawa [4] showed that if

$$
\frac{B_{0} B_{1}}{m_{0}}\left[\left(\left|A u_{0}\right|^{2}+\frac{1}{m_{0}}\left|A^{1 / 2} u_{1}\right|^{2}\right)^{1 / 2}+\frac{1}{\sqrt{m_{0}}} \int_{0}^{\infty}\left|A^{1 / 2} f(t)\right| d t\right]<\delta
$$

then there exists a unique global solution $u(t)$ to the problem (2.3)-(2.5). Here

$$
\begin{aligned}
& B_{0}=\sqrt{2} E(0)^{1 / 2}+\int_{0}^{\infty}|f(s)| d s, \\
& B_{1}=\max \left\{\left|M^{\prime}(s)\right|: 0 \leq s \leq B_{0}^{2} / m_{0}\right\} .
\end{aligned}
$$

For the regularity of solutions to the problem (2.3)-(2.5), we have:

THEOREM 2.2. Let $l \in \mathbb{N}$ be arbitrary and fixed. If in addition to the hypothesis of Theorem 2.1, $\left(u_{0}, u_{1}, f\right) \in W_{l+1} \times W_{l} \times\left(L^{1}\left(0, \infty ; W_{l}\right) \cap L^{\infty}\left(0, \infty ; W_{l-1}\right)\right)$, then the solution $u(t)$ to the problem (2.3)-(2.5) has the following properties: 
(i) $u \in L^{\infty}\left(0, \infty ; W_{l+1}\right) \cap B C\left([0, \infty) ; W_{l}\right)$.

(ii) $u^{\prime} \in L^{\infty}\left(0, \infty ; W_{l}\right) \cap B C\left([0, \infty) ; W_{l-1}\right)$.

(iii) $u^{\prime \prime} \in L^{\infty}\left(0, \infty ; W_{l-1}\right)$.

As a direct result of Theorem 2.2 , if $f$ is continuous on $[0, \infty)$, then we obtain the following.

COROLLARY 2.1. Let $u_{0} \in W_{l+1}$ and $u_{1} \in W_{l}$. If in addition to the hypothesis of Theorem 2.2, $f \in C([0, \infty) ; H)$, then the solution $u(t)$ to the problem (2.3)-(2.5) satisfies $u \in C\left([0, \infty) ; W_{l+1}\right) \cap C^{1}\left([0, \infty) ; W_{l}\right) \cap C^{2}\left([0, \infty) ; W_{l-1}\right),(l \geq 1)$.

REMARK 2.2. In Corollary 2.1 , when $l=1$, we immediately obtain the solution $u(t)$ such that $u \in C([0, \infty) ; V) \cap C^{1}([0, \infty) ; W) \cap C^{2}([0, \infty) ; H)$.

Generalizing the above results, we can consider the following nonlinear evolution equations of the form (see [8])

$$
\begin{aligned}
& u^{\prime \prime}(t)+M\left(\left|A^{k / 2} u(t)\right|^{2}\right) A^{k} u(t)+\delta u^{\prime}(t)=f(t) \text { in } H \\
& u(t) \in V_{k}\left(:=D\left(A^{k}\right)\right) \quad \text { for any } t \in[0, \infty) ; \\
& u(0)=u_{0} \in D\left(A^{k}\right), \quad u^{\prime}(0)=u_{1} \in W_{k}\left(:=D\left(A^{k / 2}\right)\right) .
\end{aligned}
$$

We let $D_{k}(\gamma):=\left\{\left(u_{0}, u_{1}, f\right) ;\left|u_{0}\right|_{v_{k}} \leq \gamma,\left|u_{1}\right|_{w_{k}} \leq \gamma, \int_{0}^{\infty}|f(t)|_{w_{k}} d t \leq \gamma\right\}$. The following results (Theorem 2.3 and Theorem 2.4) may be obtained using a similar approach as in the proof of Theorem 2.1 and Theorem 2.2.

THEOREM 2.3. Let assumptions (A.1) and (A.2) be satisfied, and $\left(u_{0}, u_{1}, f\right) \in$ $V_{k} \times W_{k} \times\left(\left(L^{1}\left(0, \infty ; W_{k}\right) \cap L^{\infty}\left(0, \infty ; W_{k-1}\right)\right)\right.$. Then there exists $\gamma_{0}(>0)$ satisfying the following. If $\left(u_{0}, u_{1}, f\right) \in D_{k}\left(\gamma_{0}\right)$, then there exists a unique solution $u(t)$ on $[0, \infty)$ to the problem $(2.11)-(2.13)$ such that

(i) $u \in L^{\infty}\left(0, \infty ; V_{k}\right) \cap B C\left([0, \infty) ; W_{k}\right), u^{\prime} \in L^{\infty}\left(0, \infty ; W_{k}\right) \cap B C\left([0, \infty) ; W_{k-1}\right)$, $u^{\prime \prime} \in L^{\infty}\left(0, \infty ; W_{k-1}\right)$;

(ii) $u$ satisfies $(2.3)$ on $[0, \infty)$;

(iii) $u(0)=u_{0}$ and $u^{\prime}(0)=u_{1}$.

THEOREM 2.4. Let $l \in \mathbb{N}$ be arbitrary and fixed. If in addition to the hypothesis of Theorem 2.3, $u_{0} \in W_{k(l+1)}, u_{1} \in W_{k l}$, and $f \in L^{1}\left(0, \infty ; W_{k l}\right) \cap L^{\infty}\left(0, \infty ; W_{k(l-1)}\right)$, then the solution $u(t)$ to the problem (2.11)-(2.13) has the following properties:

(i) $u \in L^{\infty}\left(0, \infty ; W_{k(l+1)}\right) \cap B C\left([0, \infty) ; W_{k l}\right)$.

(ii) $u^{\prime} \in L^{\infty}\left(0, \infty ; W_{k l}\right) \cap B C\left([0, \infty) ; W_{k(l-1)}\right)$.

(iii) $u^{\prime \prime} \in L^{\infty}\left(0, \infty ; W_{k(l-1)}\right)$.

Here $V_{0}=W_{0}=H$. 


\section{Preliminaries}

In this section we state some useful facts about Yosida approximations of nonnegative selfadjoint operators.

Define the Yosida approximation $A_{\lambda}$ of $A$ by $A_{\lambda}=\lambda^{-1}\left(I-J_{\lambda}\right)=A J_{\lambda}$ for $\lambda>0$, where $J_{\lambda}=(I+\lambda A)^{-1}$ and $I$ is the identity on $H$. Then it is well known that $\left\|A_{\lambda}\right\| \leq 1 / \lambda(\lambda>0), J_{\lambda} \rightarrow I$ strongly as $\lambda \rightarrow 0$ and so $A_{\lambda} u \rightarrow A u(\lambda \rightarrow 0)$ for $u \in D(A)$. Next we consider the power of degree $1 / 2$ of $A_{\lambda}$ :

$$
A_{\lambda}^{1 / 2}=A^{1 / 2} J_{\lambda}^{1 / 2} \quad(\lambda>0),
$$

where $J_{\lambda}^{1 / 2}=(I+\lambda A)^{-1 / 2}$ and $A^{1 / 2}$ is the power of degree $1 / 2$ of $A$ (see [11]). We obtain several basic properties of the operators $J_{\lambda}^{1 / 2}$ and $A_{\lambda}^{1 / 2}$.

LEMMA 3.1. Let $k \in \mathbb{N}$ be a fixed number and $\lambda>0$. Then for $1 \leq j \leq k$,

(i) $\left\|J_{\lambda}^{j / 2}\right\| \leq 1$ and $\left|A_{\lambda}^{j / 2} u\right| \leq\left|A^{j / 2} u\right|$ for $u \in D\left(A^{j / 2}\right)$;

(ii) $\left\|A_{\lambda}^{j / 2}\right\| \leq \lambda^{-j / 2}$;

(iii) $\left|u-J_{\lambda}^{j / 2} u\right| \leq j \lambda^{1 / 2}\left|A^{1 / 2} u\right|, u \in H$.

Moreover,

(iv) $\left|u-J_{\lambda}^{j} u\right| \leq j \lambda|A u|, u \in H$.

Here $A_{\lambda}^{\gamma}$ is the power of degree $\gamma$ of $A_{\lambda}$.

PROOF. Since (i) can be easily shown by the definition of $J_{\lambda}^{j / 2}$ and $A_{\lambda}^{j / 2}$, we will only prove (ii), (iii), and (iv).

Let $u \in D\left(A^{j / 2}\right)$ for $1 \leq j \leq k$. Then we have

$$
\left|\lambda^{j / 2} A^{j / 2} u\right|^{2}=\left(\lambda^{j} A^{j} u, u\right) \leq\left((I+\lambda A)^{j} u, u\right)=\left|(I+\lambda A)^{j / 2} u\right|^{2},
$$

which implies (ii).

Now we shall prove (iii). Note that (iv) can be proved similarly (use $\left|u-J_{\lambda} u\right|=$ $\left.\lambda\left|A_{\lambda} u\right|, u \in H\right)$. First, note that

$$
\left|u-J_{\lambda}^{1 / 2} u\right| \leq \lambda^{1 / 2}\left|A_{\lambda}^{1 / 2} u\right|, \quad u \in H(\text { see [4]). }
$$

From (i) and (3.2), we obtain for $1 \leq j \leq k$,

$$
\left|u-J_{\lambda}^{j / 2} u\right| \leq j\left|u-J_{\lambda}^{1 / 2} u\right| \leq j \lambda^{1 / 2}\left|A^{1 / 2} u\right|,
$$

which completes our proof.

Next we introduce the Bihari-type inequality without proof (see [5]).

LEMMA 3.2. Let $F$ and $G$ be nonnegative continuous functions on $[0, T],(T>0)$. If $[F(t)]^{2} \leq C+\int_{0}^{t} F(s) G(s) d s$ on $[0, T]$, then $F(t) \leq C^{1 / 2}+\frac{1}{2} \int_{0}^{t} G(s) d s$ on $[0, T]$, where $C$ is a positive constant. 


\section{Proof of theorems}

4.1. Proof of Theorem 2.1 (Existence and Uniqueness). In this section we shall prove Theorem 2.1 using the Yosida approximation. Throughout this section we let $\lambda>0$ be any number and $A_{\lambda}$ be the Yosida approximation of $A$.

First we consider the approximate problem of the following differential equation by applying the Yosida approximation

$$
\begin{aligned}
& u_{\lambda}^{\prime \prime}(t)+M\left(\left|A_{\lambda}^{1 / 2} u_{\lambda}(t)\right|^{2}\right) A_{\lambda} u_{\lambda}(t)+\delta u_{\lambda}^{\prime}(t)=f(t), \\
& u_{\lambda}(0)=u_{0} \in V, \quad u_{\lambda}^{\prime}(0)=u_{1} \in W .
\end{aligned}
$$

Using the mean value theorem for $M(t)$, we can easily show that the mapping $u \rightarrow$ $M\left(\left|A_{\lambda}^{1 / 2} u\right|^{2}\right) A_{\lambda} u$ is locally Lipschitz continuous for each $\lambda$. Then it is well known that the problem (4.1)-(4.2) has a unique local approximate solution $u_{\lambda} \in C^{\prime}\left(\left[0, T_{\lambda}\right) ; H\right)$ on some interval $\left[0, T_{\lambda}\right)$ and moreover, $u_{\lambda}^{\prime}(t)$ is absolutely continuous and (4.1) holds a.e. on $\left[0, T_{\lambda}\right)$ (see $\left.[4]\right)$.

Now we shall see that $u_{\lambda}(t)$ can be extended to $[0, \infty)$. To see this, we need $a$ priori estimates for the solution $u_{\lambda}(t)$.

\section{A priori estimates}

PROPOSITION 4.1. If $u_{0} \in V$ and $u_{1} \in W$, then there exists a positive constant $M_{1}$, which does not depend on $\lambda$ such that

$$
\left|u_{\lambda}(t)\right| \leq M_{1} \text { on }\left[0, T_{\lambda}\right)
$$

We need the following lemma in order to prove this result. In fact, this lemma is shown by applying energy methods to the problem (4.1)-(4.2).

LEMMA 4.1. Let $u_{0} \in V$ and $u_{1} \in W$. Then the following inequality holds on $\left[0, T_{\lambda}\right)$

$$
\sup _{i \in\left[0, T_{\lambda}\right)}\left\{\left|u_{\lambda}^{\prime}(t)\right|^{2}, m_{0}\left|A_{\lambda}^{1 / 2} u_{\lambda}(t)\right|^{2}, 2 \delta \int_{0}^{t}\left|u_{\lambda}^{\prime}(s)\right|^{2} d s\right\} \leq B_{0}^{2},
$$

where $B_{0}$ is given by (2.9) and $m_{0}$ is the constant given in assumption (A.2).

PROOF. If we multiply (4.1) by $2 u_{\lambda}^{\prime}(t)$, then we obtain a.e. on [0, $\left.T_{\lambda}\right)$,

$$
\frac{d\left|u_{\lambda}^{\prime}(t)\right|^{2}}{d t}+M\left(\left|A_{\lambda}^{1 / 2} u_{\lambda}(t)\right|^{2}\right) \frac{d}{d t}\left|A_{\lambda}^{1 / 2} u_{\lambda}(t)\right|^{2}+2 \delta\left|u_{\lambda}^{\prime}(t)\right|^{2}=2\left(f(t), u_{\lambda}^{\prime}(t)\right) .
$$

Integrating (4.5) on $(0, t), t \in\left[0, T_{\lambda}\right)$ and using (2.6), and (2.7) we have

$$
\left|u_{\lambda}^{\prime}(t)\right|^{2}+m_{0}\left|A_{\lambda}^{1 / 2} u_{\lambda}(t)\right|^{2}+2 \delta \int_{0}^{t}\left|u_{\lambda}^{\prime}(s)\right|^{2} d s \leq 2 E(0)+2 \int_{0}^{t}|f(t)|\left|u_{\lambda}^{\prime}(s)\right| d s .
$$


If we set

$$
F(t):=\left[\left|u_{\lambda}^{\prime}(t)\right|^{2}+m_{0}\left|A_{\lambda}^{1 / 2} u_{\lambda}(t)\right|^{2}+2 \delta \int_{0}^{t}\left|u_{\lambda}^{\prime}(s)\right|^{2} d s\right]^{1 / 2},
$$

then (4.6) implies

$$
[F(t)]^{2} \leq 2 E(0)+2 \int_{0}^{t}|f(s)| F(s) d s
$$

Therefore, the desired result follows by applying Lemma 3.2 to (4.7).

PROOF OF PROPOSITION 4.1. If we multiply (4.1) by $u_{\lambda}(t)$, then we have a.e. on $\left[0, T_{\lambda}\right)$,

$$
\begin{aligned}
& \frac{d}{d t}\left(u_{\lambda}^{\prime}(t), u_{\lambda}(t)\right)-\left|u_{\lambda}^{\prime}(t)\right|^{2}+M\left(\left|A_{\lambda}^{1 / 2} u_{\lambda}(t)\right|^{2}\right)\left|A_{\lambda}^{1 / 2} u_{\lambda}(t)\right|^{2}+\frac{\delta}{2} \frac{d}{d t}\left|u_{\lambda}(t)\right|^{2} \\
& \quad=\left(f(t), u_{\lambda}(t)\right) .
\end{aligned}
$$

Integrating (4.8) on $(0, t), t \in\left[0, T_{\lambda}\right)$ and using the Schwarz inequality, we obtain

$$
\begin{aligned}
& \frac{\delta}{2}\left|u_{\lambda}(t)\right|^{2}+\int_{0}^{t} M\left(\left|A_{\lambda}^{1 / 2} u_{\lambda}(s)\right|^{2}\right)\left|A_{\lambda}^{1 / 2} u_{\lambda}(s)\right|^{2} d s \\
& \quad \leq \frac{\delta}{2}\left|u_{0}\right|^{2}+\left|u_{0}\right|\left|u_{1}\right|+\left|u_{\lambda}^{\prime}(t)\right|\left|u_{\lambda}(t)\right|+\int_{0}^{t}\left|u_{\lambda}^{\prime}(s)\right|^{2} d s+\int_{0}^{t}|f(s)|\left|u_{\lambda}(s)\right| d s .
\end{aligned}
$$

Using the inequality $\left|u_{\lambda}^{\prime}(t)\right|\left|u_{\lambda}(t)\right| \leq \frac{\delta}{4}\left|u_{\lambda}(t)\right|^{2}+\frac{1}{\delta}\left|u_{\lambda}^{\prime}(t)\right|^{2}$, we obtain from (4.9)

$$
\frac{\delta}{4}\left|u_{\lambda}(t)\right|^{2} \leq \frac{\delta}{2}\left|u_{0}\right|^{2}+\left|u_{0}\right|\left|u_{1}\right|+\frac{1}{\delta}\left|u_{\lambda}^{\prime}(t)\right|^{2}+\int_{0}^{t}\left|u_{\lambda}^{\prime}(s)\right|^{2} d s+\int_{0}^{t}|f(s)|\left|u_{\lambda}(s)\right| d s .
$$

From the last inequality and (4.4), we can see that

$$
\left|u_{\lambda}(t)\right|^{2} \leq M_{1}(\delta)+\frac{4}{\delta} \int_{0}^{t}|f(s)|\left|u_{\lambda}(s)\right| d s,
$$

where $M_{1}(\delta):=2\left|u_{0}\right|^{2}+(4 / \delta)\left|u_{0}\right|\left|u_{1}\right|+6 B_{0}^{2} / \delta^{2}$. Therefore (4.3) follows from Lemma 3.2.

From Proposition 4.1, it follows that $u_{\lambda}(t)$ is uniformly bounded, hence can be extended to $[0, \infty)$.

Now we prove that $u_{\lambda}(t)$ and $u_{\lambda}^{\prime}(t)$ are uniformly bounded in $V$ and $W$, respectively.

Proposition 4.2. Let $\left(u_{0}, u_{1}\right) \in V \times W$. Then there exists $\gamma_{0}>0$ such that if (2.8) is satisfied, then there exists a positive constant $M_{2}$, which does not depend on $\gamma_{0}$ such that $\sup _{t \in[0, \infty)}\left\{\left|A_{\lambda} u_{\lambda}(t)\right|,\left|A_{\lambda}^{1 / 2} u_{\lambda}^{\prime}(t)\right|\right\} \leq M_{2}$. 
PrOOF. If we multiply $(4.1)$ by $2 A_{\lambda} u_{\lambda}^{\prime}(t)$, then we obtain a.e. on $[0, \infty)$

$$
\begin{gathered}
\frac{d}{d t}\left\{\left|A_{\lambda}^{1 / 2} u_{\lambda}^{\prime}(t)\right|^{2}+M\left(\left|A_{\lambda}^{1 / 2} u_{\lambda}(t)\right|^{2}\right)\left|A_{\lambda} u_{\lambda}(t)\right|^{2}\right\}+2 \delta\left|A_{\lambda}^{1 / 2} u_{\lambda}^{\prime}(t)\right|^{2} \\
=2\left(A_{\lambda}^{1 / 2} f(t), A_{\lambda}^{1 / 2} u_{\lambda}^{\prime}(t)\right) \\
\quad+2\left|A_{\lambda} u_{\lambda}(t)\right|^{2} M^{\prime}\left(\left|A_{\lambda}^{1 / 2} u_{\lambda}(t)\right|^{2}\left(A_{\lambda}^{1 / 2} u_{\lambda}(t), A_{\lambda}^{1 / 2} u_{\lambda}(t)\right) .\right.
\end{gathered}
$$

Integrating (4.10) on $(0, t), t \in[0, \infty)$, we have

$$
\begin{aligned}
\left|A_{\lambda}^{1 / 2} u_{\lambda}^{\prime}(t)\right|^{2}+M\left(\left|A_{\lambda}^{1 / 2} u_{\lambda}(t)\right|^{2}\right)\left|A_{\lambda} u_{\lambda}(t)\right|^{2}+2 \delta \int_{0}^{t}\left|A_{\lambda}^{1 / 2} u_{\lambda}^{\prime}(s)\right|^{2} d s \\
=\left|A^{1 / 2} u_{1}\right|^{2}+M\left(\left|A^{1 / 2} u_{0}\right|^{2}\right)\left|A u_{0}\right|^{2}+2 \int_{0}^{t}\left(A_{\lambda}^{1 / 2} f(s), A_{\lambda}^{1 / 2} u_{\lambda}^{\prime}(s)\right) d s \\
\quad+2 \int_{0}^{t} M^{\prime}\left(\left|A_{\lambda}^{1 / 2} u_{\lambda}(s)\right|^{2}\right)\left(A_{\lambda}^{1 / 2} u_{\lambda}^{\prime}(s), A_{\lambda}^{1 / 2} u_{\lambda}(s)\right)\left|A_{\lambda} u_{\lambda}(s)\right|^{2} d s .
\end{aligned}
$$

Using the Schwarz inequality, the assumption (A.2), (4.4) and (4.11), we get

$$
\begin{aligned}
& \left|A_{\lambda}^{1 / 2} u_{\lambda}^{\prime}(t)\right|^{2}+m_{0}\left|A_{\lambda} u_{\lambda}(t)\right|^{2}+2 \delta \int_{0}^{t}\left|A_{\lambda}^{1 / 2} u_{\lambda}^{\prime}(s)\right|^{2} d s \\
& \leq\left.|| A^{1 / 2} u_{1}\right|^{2}+B_{2}\left|A u_{0}\right|^{2}+2 \int_{0}^{t}\left|A_{\lambda}^{1 / 2} f(s)\right|\left|A_{\lambda}^{1 / 2} u_{\lambda}^{\prime}(s)\right| d s \\
& \quad+2 \frac{B_{0} B_{1}}{\sqrt{m_{0}}} \int_{0}^{t}\left|A_{\lambda}^{1 / 2} u_{\lambda}^{\prime}(s)\right|\left|A_{\lambda} u_{\lambda}(s)\right|^{2} d s,
\end{aligned}
$$

where $B_{i}, i=0,1$, are the constants given by (2.9) and (2.10), respectively and $B_{2}$ is given by

$$
B_{2}:=\max \left\{|M(s)|: 0 \leq s \leq B_{0}^{2} / m_{0}\right\} .
$$

If we multiply (4.1) by $A_{\lambda} u_{\lambda}(t)$, then we have a.e. in $[0, \infty)$

$$
\begin{aligned}
& \frac{d}{d t}\left[\left(A_{\lambda}^{1 / 2} u_{\lambda}^{\prime}(t), A_{\lambda}^{1 / 2} u_{\lambda}(t)\right)+\frac{\delta}{2}\left|A_{\lambda}^{1 / 2} u_{\lambda}(t)\right|^{2}\right\}+M\left(\left|A_{\lambda}^{1 / 2} u_{\lambda}(t)\right|^{2}\right)\left|A_{\lambda} u_{\lambda}(t)\right|^{2} \\
& \quad=\left|A_{\lambda}^{1 / 2} u_{\lambda}^{\prime}(t)\right|^{2}+\left(A_{\lambda}^{1 / 2} f(t), A_{\lambda}^{1 / 2} u_{\lambda}(t)\right) .
\end{aligned}
$$

Integrating (4.14) on $(0, t), t \in[0, \infty)$ and then using (A.2), we obtain

$$
\begin{aligned}
-\left|A_{\lambda}^{1 / 2} u_{\lambda}^{\prime}(t)\right|\left|A_{\lambda}^{1 / 2} u_{\lambda}(t)\right|+\frac{\delta}{2}\left|A_{\lambda}^{1 / 2} u_{\lambda}(t)\right|^{2}+m_{0} \int_{0}^{t}\left|A_{\lambda} u_{\lambda}(s)\right|^{2} d s \\
\leq\left|A^{1 / 2} u_{1}\right|\left|A^{1 / 2} u_{0}\right|+\frac{\delta}{2}\left|A^{1 / 2} u_{0}\right|^{2}+\int_{0}^{t}\left|A_{\lambda}^{1 / 2} u_{\lambda}^{\prime}(s)\right|^{2} d s \\
\quad+\int_{0}^{t}\left|A_{\lambda}^{1 / 2} f(s)\right|\left|A_{\lambda}^{1 / 2} u_{\lambda}(s)\right| d s .
\end{aligned}
$$


We now assume that the data $\left(u_{0}, u_{1}, f\right) \in D(\gamma)$.

Multiplying (4.15) by $\delta$ and adding it to (4.12), we obtain

$$
\begin{gathered}
\frac{1}{2}\left|A_{\lambda}^{1 / 2} u_{\lambda}^{\prime}(t)\right|^{2}+\frac{1}{2}\left(\left|A_{\lambda}^{1 / 2} u_{\lambda}^{\prime}(t)\right|-\delta\left|A_{\lambda}^{1 / 2} u_{\lambda}(t)\right|\right)^{2}+\delta \int_{0}^{t}\left|A_{\lambda}^{1 / 2} u_{\lambda}^{\prime}(s)\right|^{2} d s \\
+m_{0}\left|A_{\lambda} u_{\lambda}(t)\right|^{2}+\int_{0}^{t}\left(m_{0} \delta-2 \frac{B_{0} B_{1}}{\sqrt{m_{0}}}\left|A_{\lambda}^{1 / 2} u_{\lambda}^{\prime}(s)\right|\right)\left|A_{\lambda} u_{\lambda}(s)\right|^{2} d s \\
\leq\left|A^{1 / 2} u_{1}\right|^{2}+B_{2}\left|A u_{0}\right|^{2}+\delta\left|A^{1 / 2} u_{1}\right|\left|A^{1 / 2} u_{0}\right|+\frac{\delta^{2}}{2}\left|A^{1 / 2} u_{0}\right|^{2} \\
\quad+2 \int_{0}^{t}\left|A_{\lambda}^{1 / 2} f(s)\right|\left|A_{\lambda}^{1 / 2} u_{\lambda}^{\prime}(s)\right| d s+\frac{\delta B_{0}}{\sqrt{m_{0}}} \int_{0}^{t}\left|A_{\lambda}^{1 / 2} f(s)\right| d s \\
\leq L(\gamma)+2 \int_{0}^{t}\left|A_{\lambda}^{1 / 2} f(s)\right|\left|A_{\lambda}^{1 / 2} u_{\lambda}^{\prime}(s)\right| d s,
\end{gathered}
$$

where $L(\gamma):=\gamma^{2}\left(1+B_{2}+\delta+\delta^{2} / 2\right)+\left(\delta B_{0} / \sqrt{m_{0}}\right) \gamma$. Once if we assume that the inequality

$$
m_{0} \delta-2 \frac{B_{0} B_{1}}{\sqrt{m_{0}}}\left|A_{\lambda}^{1 / 2} u_{\lambda}^{\prime}(t)\right| \geq 0 \text { on }[0, \infty),
$$

holds, then we can obtain using (4.16) and Lemma 3.2

$$
\begin{aligned}
\left|A_{\lambda}^{1 / 2} u_{\lambda}^{\prime}(t)\right|^{2}+2 m_{0}\left|A_{\lambda} u_{\lambda}(t)\right|^{2} & \leq \sqrt{2} L(\gamma)^{1 / 2}+2 \int_{0}^{t}\left|A_{\lambda}^{1 / 2} f(s)\right| d s \\
& \leq \tilde{L}(\gamma) \quad\left(:=\sqrt{2} L(\gamma)^{1 / 2}+2 \gamma\right) .
\end{aligned}
$$

Noting that $\tilde{L}(\cdot)$ is an increasing function of $\gamma$ and $\tilde{L}(0)=0$, we can choose $\gamma_{0}>0$ such that $\sqrt{\tilde{L}\left(\gamma_{0}\right)} \leq m_{0}^{3 / 2} \delta /\left(2 B_{0} B_{1}\right)$. Hence it says that $\left|A_{\lambda}^{1 / 2} u_{\lambda}^{\prime}(t)\right| \leq \sqrt{\tilde{L}\left(\gamma_{0}\right)} \leq$ $m_{0}^{3 / 2} \delta /\left(2 B_{0} B_{1}\right)$, that is, (4.17) is satisfied. Consequently, for $\left(u_{0}, u_{1}, f\right) \in D\left(\gamma_{0}\right)$, (4.17) is verified, which completes our proof.

Proposition 4.3. Let $u_{0} \in V$ and $u_{1} \in W$. If (2.8) is satisfied, then there exists a positive constant $M_{3}$, which does not depend on $\lambda$ such that $\left|u_{\lambda}^{\prime \prime}(t)\right| \leq M_{3}$ on $[0, \infty)$.

PROOF. If we multiply (4.1) by $u_{\lambda}^{\prime \prime}(t)$, then we have a.e. on $[0, \infty)$

$$
\left|u_{\lambda}^{\prime \prime}(t)\right|^{2}+\left(M\left(\left|A_{\lambda}^{1 / 2} u_{\lambda}(t)\right|^{2}\right) A_{\lambda} u_{\lambda}(t)+\delta u_{\lambda}^{\prime}(t)-f(t), u_{\lambda}^{\prime \prime}(t)\right)=0 .
$$

Using the Schwarz inequality and Proposition 4.2, we obtain by (4.13) and (4.4),

$$
\left|u_{\lambda}^{\prime \prime}(t)\right| \leq B_{2} M_{2}+\delta B_{0}+\operatorname{ess} \sup \{|f(s)|: 0 \leq s<\infty\},
$$

where $B_{i}(i=0,2)$ and $M_{2}$ are constants given by (2.9), (4.13) and in Proposition 4.2, respectively. This completes our proof. 
As a direct result of Proposition 4.2 and Proposition 4.3, we have the following:

COROLlary 4.1. Let $u_{0} \in V$ and $u_{1} \in W$. Assume that (2.8) is satisfied. Then for any $\lambda>0,\left\{u_{\lambda}(\cdot)\right\}_{\lambda},\left\{u_{\lambda}^{\prime}(\cdot)\right\}_{\lambda}$, and $\left\{u_{\lambda}^{\prime \prime}(\cdot)\right\}_{\lambda}$ are bounded in $L^{\infty}(0, \infty ; V), L^{\infty}(0, \infty ; W)$, and $L^{\infty}(0, \infty ; H)$, respectively.

\section{Passage to the limit}

In this section we establish the uniform convergence of solutions to the problem (2.3)-(2.5) on finite intervals of arbitrary length as $\lambda \rightarrow 0$. In what follows we will let $T>0$ be arbitrary and let $\left\{\lambda_{n}\right\}_{n}$ be a sequence such that $\lambda_{n}>0(n \in \mathbb{N})$ and $\lambda_{n} \rightarrow 0$ as $n \rightarrow \infty$.

LEMMA 4.2. If for any $\lambda>0, u_{\lambda}(\cdot)$ is a solution to the problem (4.1)-(4.2), then there exist a subsequence $\left\{u_{\lambda_{n}}(\cdot)\right\}_{n}$ of $\left\{u_{\lambda}(\cdot)\right\}_{\lambda}$ and $u(\cdot) \in B C([0, \infty) ; H)$ such that

$$
u_{\lambda_{n}}(\cdot) \rightarrow u(\cdot) \text { in } C([0, T] ; H) \text { as } n \rightarrow \infty .
$$

Moreover, if (2.8) is satisfied, then there is a subsequence $\left\{u_{\mu_{n}}(\cdot)\right\}_{n}$ of $\left\{u_{\lambda_{n}}(\cdot)\right\}_{n}$ and $u^{\prime}(\cdot) \in B C([0, \infty) ; H)$ such that

$$
u_{\mu_{n}}^{\prime}(\cdot) \rightarrow u^{\prime}(\cdot) \text { in } C([0, T] ; H) \text { as } n \rightarrow \infty .
$$

Here the convergence is uniform with respect to $t \in[0, T]$.

PRoof. First we show that for any $t \in[0, \infty),\left\{J_{\lambda}^{1 / 2} u_{\lambda}(t)\right\}_{\lambda}$ is precompact in $H$. In fact, we have for any $t \in[0, \infty)$,

$$
\begin{aligned}
& \left|J_{\lambda}^{1 / 2} u_{\lambda}(t)-J_{\mu}^{1 / 2} u_{\mu}(t)\right|_{W} \\
& \quad=\left|J_{\lambda}^{1 / 2} u_{\lambda}(t)-J_{\mu}^{1 / 2} u_{\mu}(t)\right|+\left|A_{\lambda}^{1 / 2} u_{\lambda}(t)-A_{\mu}^{1 / 2} u_{\mu}(t)\right| \\
& \quad \leq\left|u_{\lambda}(t)\right|+\left|u_{\mu}(t)\right|+\left|A_{\lambda}^{1 / 2} u_{\lambda}(t)\right|+\left|A_{\mu}^{1 / 2} u_{\mu}(t)\right|,
\end{aligned}
$$

where $\lambda>0$ and $\mu>0$ are arbitrary.

From (4.3), (4.4), and the definition of $A_{\lambda}^{1 / 2},(4.21)$ implies that for any $t \in[0, \infty)$, $\left\{J_{\lambda}^{1 / 2} u_{\lambda}(t)\right\}_{\lambda}$ is bounded in $W$. Thus we see from the assumption (A.1) that for any $t \in[0, \infty),\left\{J_{\lambda}^{1 / 2} u_{\lambda}(t)\right\}_{\lambda}$ is precompact in $H$. Moreover, from Lemma 3.1 (i) and (4.4), we easily observe that $\left\{J_{\lambda}^{1 / 2} u_{\lambda}(\cdot)\right\}_{\lambda}$ is equicontinuous. Hence, applying the ArzelàAscoli theorem to $\left\{J_{\lambda}^{1 / 2} u_{\lambda}(\cdot)\right\}_{\lambda}$ in $C([0, T] ; H)$, we find a subsequence $\left\{J_{\lambda_{n}}^{1 / 2} u_{\lambda_{n}}(\cdot)\right\}_{\lambda}$ and $u(\cdot) \in B C([0, \infty) ; H)$ such that

$$
J_{\lambda_{n}}^{1 / 2} u_{\lambda_{n}}(\cdot) \rightarrow u(\cdot) \quad \text { in } C([0, T] ; H) \quad \text { as } n \rightarrow \infty .
$$

Noting that $\left|u_{\lambda_{n}}(t)-J_{\lambda_{n}}^{1 / 2} u_{\lambda_{n}}(t)\right| \leq \lambda_{n}^{1 / 2}\left|A_{\lambda_{n}}^{1 / 2} u_{\lambda_{n}}(t)\right|$ (see Lemma 3.1 (iii)), we observe that $u_{\lambda_{n}}(\cdot) \rightarrow u(\cdot)$ in $C([0, T] ; H)$ as $n \rightarrow \infty$.

Noting that $J_{\lambda_{n}}^{1 / 2} u_{\lambda_{n}}^{\prime}(\cdot)$ and $A_{\lambda}^{1 / 2} u_{\lambda_{n}}(\cdot)$ belong to $B C([0, \infty) ; H)$, we can also prove (4.20) in the same way as in the proof of (4.19). 
LEMMA 4.3. Let $u(\cdot),\left\{\lambda_{n}\right\}_{n}$, and $\left\{\mu_{n}\right\}_{n}$ be as in Lemma 4.2. Assume that (2.8) is satisfied. Then $u(\cdot) \in L^{\infty}(0, \infty ; V), u^{\prime}(\cdot) \in L^{\infty}(0, \infty ; W)$ and

$$
\begin{aligned}
A u(t) & =\text { weak } \lim _{n \rightarrow \infty} A_{\lambda_{n}} u_{\lambda_{n}}(t) \text { in } H, \\
A^{1 / 2} u^{\prime}(t) & =\text { weak } \lim _{n \rightarrow \infty} A_{\mu_{n}}^{1 / 2} u_{\mu_{n}}^{\prime}(t) \text { in } H .
\end{aligned}
$$

Moreover, $u(\cdot) \in B C([0, \infty) ; W)$ and

$$
M\left(\left|A^{1 / 2} u(t)\right|^{2}\right) A u(t)=\text { weak } \lim _{n \rightarrow \infty} M\left(\left|A_{\lambda_{n}}^{1 / 2} u_{\lambda_{n}}(t)\right|^{2}\right) A_{\lambda_{n}} u_{\lambda_{n}}(t)
$$

Here the convergence is uniform with respect to $t \in[0, T]$.

Proof. We note that $A^{\alpha}$ is weakly closed and $D\left(A^{\alpha}\right)$ is dense in $H(\alpha=1,1 / 2)$. From Proposition 4.1 and Proposition 4.2, we observe that $A_{\lambda} u_{\lambda_{n}}(\cdot)$ and $A_{\mu_{n}} u_{\mu_{n}}^{\prime}(\cdot)$ belong to $B C([0, \infty) ; H)$.

Thus (4.23) and (4.24) follow from (4.19) and (4.20). We also have

$$
\begin{aligned}
&|A u(t)| \leq \lim _{n \rightarrow \infty} \inf \left|A_{\lambda_{n}} u_{\lambda_{n}}(t)\right| \leq M_{2}, \\
&\left|A^{1 / 2} u^{\prime}(t)\right| \leq \lim _{n \rightarrow \infty} \inf \left|A_{\mu_{n}}^{1 / 2} u_{\mu_{n}}^{\prime}(t)\right| \leq M_{2} .
\end{aligned}
$$

These imply that $u(\cdot) \in L^{\infty}(0, \infty ; V)$ and $u^{\prime}(\cdot) \in L^{\infty}(0, \infty ; W)$. In order to prove (4.25), we first show that $u(\cdot) \in B C([0, \infty) ; W)$ and

$$
A_{\lambda_{n}}^{1 / 2} u_{\lambda_{n}}(\cdot) \rightarrow A^{1 / 2} u(\cdot) \quad \text { in } C([0, T] ; H) \quad \text { as } n \rightarrow \infty
$$

From the definition of $A_{\lambda_{n}}^{1 / 2}$ and using the Schwarz inequality, we observe that

$$
\begin{aligned}
\left|A_{\lambda_{n}}^{1 / 2} u_{\lambda_{n}}(t)-A^{1 / 2} u(t)\right|^{2} & =\left|A_{\lambda_{n}}^{1 / 2} u_{\lambda_{n}}(t)\right|^{2}-2\left(A u(t), J_{\lambda_{n}}^{1 / 2} u_{\lambda_{n}}(t)\right)+\left|A^{1 / 2} u(t)\right|^{2} \\
& =\left|A_{\lambda_{n}}^{1 / 2} u_{\lambda_{n}}(t)\right|^{2}-\left|A^{1 / 2} u(t)\right|^{2}+2\left(A u(t), u(t)-J_{\lambda_{n}}^{1 / 2} u_{\lambda_{n}}(t)\right) \\
& \leq\left|A_{\lambda_{n}}^{1 / 2} u_{\lambda_{n}}(t)\right|^{2}-\left|A^{1 / 2} u(t)\right|^{2}+2|A u(t)|\left|u(t)-J_{\lambda_{n}}^{1 / 2} u_{\lambda_{n}}(t)\right|
\end{aligned}
$$

Thus it suffices, by (4.22) and (4.26), to show that

$$
\left(A_{\lambda_{n}} u_{\lambda_{n}}(t), u_{\lambda_{n}}(t)\right) \rightarrow(A u(t), u(t)) \quad \text { in } C[0, T] \quad \text { as } n \rightarrow \infty
$$

Indeed, from Lemma 3.1 (iv) and using the Schwarz inequality, we have

$$
\begin{aligned}
& \left|\left(A_{\lambda_{n}} u_{\lambda_{n}}(t), u_{\lambda_{n}}(t)\right)-(A u(t), u(t))\right| \\
& \quad=\left|\left(A_{\lambda_{n}} u_{\lambda_{n}}(t)-A_{\lambda_{n}} u(t), u_{\lambda_{n}}(t)\right)+\left(J_{\lambda_{n}} u(t), A u_{\lambda_{n}}(t)\right)-(A u(t), u(t))\right| \\
& \quad=\left|\left(A_{\lambda_{n}} u_{\lambda_{n}}(t), u_{\lambda_{n}}(t)-u(t)\right)+\left(A u(t), J_{\lambda_{n}} u(t)-u(t)\right)+\left(A_{\lambda_{n}} u(t), u_{\lambda_{n}}(t)-u(t)\right)\right| \\
& \quad \leq \lambda_{n}|A u(t)|^{2}+\left(\left|A_{\lambda_{n}} u_{\lambda_{n}}(t)\right|+\left|A_{\lambda_{n}} u(t)\right|\right)\left|u_{\lambda_{n}}(t)-u(t)\right| .
\end{aligned}
$$


So (4.29) follows from (4.19) and (4.26). Hence we obtain (4.28) and we also have by (4.4)

$$
\left|A^{1 / 2} u(t)\right|=\lim _{n \rightarrow \infty}\left|A_{\lambda_{n}}^{1 / 2} u_{\lambda_{n}}(t)\right| \leq B_{0} / \sqrt{m_{0}},
$$

where $B_{0}$ is the constant given by $(2.9)$, that is, $u(\cdot) \in B C([0, \infty) ; W)$. Finally, by using the mean value theorem for $M(\cdot)$, our final assertion immediately follows from (4.4), (4.23), and (4.29).

We are now in a position to show that $u(\cdot)$, given by Lemma 4.2 , is a solution to the problem (2.3)-(2.5).

PROPOSITION 4.4. Let $u(\cdot)$ and $\left\{\mu_{n}\right\}_{n}$ be as in Lemma 4.2. Assume that (2.8) is satisfied. Then $u^{\prime \prime}(\cdot) \in L^{\infty}(0, \infty ; H)$ and

$$
u^{\prime \prime}(t)+M\left(\left|A^{1 / 2} u(t)\right|^{2}\right) A u(t)+\delta u^{\prime}(t)=f(t) \text { a.e. in } H .
$$

Proof. From Proposition 4.3, we can observe that $u^{\prime}(t)$ is Lipschitz continuous and so it is absolutely continuous. Hence $u^{\prime \prime}(t) \in L^{\infty}(0, \infty ; H)$ exists a.e. on $(0, \infty)$. We also see from (4.1), (4.20), and (4.25) that

$$
f(t)-\delta u^{\prime}(t)-M\left(\left|A^{1 / 2} u(t)\right|^{2}\right) A u(t)=\text { weak } \lim _{n \rightarrow \infty} u_{\mu_{n}}^{\prime \prime}(t) .
$$

Put $w_{n}(t):=\left(u_{\mu_{n}}^{\prime}(t)-u_{\mu_{n}}^{\prime}(s)\right) /(t-s)$ on $t \in[0, T]$. Here $s(\neq t)$ is arbitrary but fixed on $[0, T]$. Then clearly, $\lim _{t \rightarrow s} w_{n}(t)=u_{\mu_{n}}^{\prime \prime}(s)$ a.e. on $(0, \infty)$ and by virtue of (4.20), $\lim _{n \rightarrow \infty} w_{n}(t)=\left(\boldsymbol{u}^{\prime}(t)-\boldsymbol{u}^{\prime}(s)\right) /(t-s)$, uniformly on [0,T]. Hence we obtain by the continuity of $(\cdot, \cdot)$,

$$
\lim _{n \rightarrow \infty}\left(u_{\mu_{n}}^{\prime \prime}(s), v\right)=\lim _{n \rightarrow \infty}\left(\lim _{t \rightarrow s} w_{n}(t), v\right)=\left(u^{\prime \prime}(s), v\right), \quad v \in H .
$$

So, our assertion follows from (4.31) and (4.32).

\section{Uniqueness}

LEMMA 4.4. Let $u$ and $v$ be solutions to the problem (2.3)-(2.5). If $w(t) \in$ $C^{1}([0, \infty), V)$ is a solution of

$$
\begin{aligned}
& w^{\prime \prime}(t)+M\left(\left|A^{1 / 2} u(t)\right|^{2}\right) A w(t)+\delta w^{\prime}(t)=F(u(t), v(t)), \\
& w(0)=0, \quad w^{\prime}(0)=0,
\end{aligned}
$$

where $|F(u(t), v(t))| \leq M_{4}\left|A^{1 / 2} w(t)\right|$ for all $t \in[0, \infty)$ and some constant $M_{4}>0$, then $w(t) \equiv 0$ for $t \in[0, \infty)$. 
ProOF. If we multiply (4.33) by $2 w^{\prime}(t)$, we obtain

$$
\begin{aligned}
& \frac{d}{d t}\left|w^{\prime}(t)\right|^{2}+M\left(\left|A^{1 / 2} u(t)\right|^{2}\right) \frac{d}{d t}\left|A^{1 / 2} w(t)\right|^{2}+2 \delta\left|w^{\prime}(t)\right|^{2} \\
& =2\left(F(u(t), v(t)), w^{\prime}(t)\right) .
\end{aligned}
$$

Integrating (4.34) on $(0, t), t \in[0, \infty)$ and using the Schwarz inequality, we obtain

$$
\begin{aligned}
& \left|w^{\prime}(t)\right|^{2}+M\left(\left|A^{1 / 2} u(t)\right|^{2}\right)\left|A^{1 / 2} w(t)\right|^{2}+2 \delta \int_{0}^{t}\left|w^{\prime}(s)\right|^{2} d s \\
& \quad \leq \int_{0}^{t}\left|\frac{d}{d t} M\left(\left|A^{1 / 2} u(s)\right|^{2}\right)\right|\left|A^{1 / 2} w(s)\right|^{2} d s+2 \int_{0}^{t}|F(u(s), v(s))|\left|w^{\prime}(s)\right| d s .
\end{aligned}
$$

From (A.2), (4.27), and (4.30), this inequality yields

$$
\left|w^{\prime}(t)\right|^{2}+m_{0}\left|A^{1 / 2} w(t)\right|^{2} \leq\left(\frac{2 B_{0} B_{1} M_{2}}{m_{0} \sqrt{m_{0}}}+\frac{M_{4}}{\sqrt{m_{0}}}\right) \int_{0}^{t}\left(\left|w^{\prime}(s)\right|^{2}+m_{0}\left|A^{1 / 2} w(s)\right|^{2}\right) d s .
$$

Therefore we obtain $w \equiv 0$ by the Gronwall inequality (see [5]).

PROPOSITION 4.5. Assume that (2.8) is satisfied. Then the problem (2.3)-(2.5) has a unique solution. In fact, $u(\cdot)$ in Lemma 4.2 is the solution of the problem (2.3)-(2.5).

Proof. Let $u(\cdot)$ be as in Lemma 4.2. Then from Lemma 4.2 and Lemma 4.3, Proposition 4.4, and Proposition (4.2), clearly $u(\cdot)$ satisfies the problem (2.3)-(2.5). Thus it remains to show the uniqueness of the solution. Let $u$ and $v$ be two solutions to the problem (2.3)-(2.5). Then $w=u-v$ satisfies

$$
\begin{aligned}
w^{\prime \prime}(t) & +M\left(\left|A^{1 / 2} u(t)\right|^{2}\right) A w(t)+\delta w^{\prime}(t) \\
& =-\left\{M\left(\left|A^{1 / 2} u(t)\right|^{2}\right)-M\left(\left|A^{1 / 2} v(t)\right|^{2}\right)\right\} A v(t), \\
w(0) & =0, \quad w^{\prime}(0)=0 .
\end{aligned}
$$

Moreover, by the mean value theorem for $M(\cdot)$ and (4.30), we have

$$
\begin{aligned}
& \left|\left(M\left(\left|A^{1 / 2} u(t)\right|^{2}\right)-M\left(\left|A^{1 / 2} v(t)\right|^{2}\right)\right) A v(t)\right| \\
& \quad \leq B_{1}\left(\left|A^{1 / 2} u(t)\right|+\left|A^{1 / 2} v(t)\right|\right)\left|A^{1 / 2} w(t)\right||A v(t)| \leq 2 \frac{B_{0} M_{2} B_{1}}{\sqrt{m_{0}}}\left|A^{1 / 2} w(t)\right| .
\end{aligned}
$$

So, uniqueness follows from Lemma 4.4. 
4.2. Proof of Theorem 2.2 (Regularity). We consider the initial value problem

$$
\begin{aligned}
& u^{\prime \prime}(t)+M\left(\left|A^{1 / 2} u(t)\right|^{2}\right) A u(t)+\delta u^{\prime}(t)=f(t) \text { in } H, \\
& u(0)=u_{0} \in W_{l+1}, \quad u^{\prime}(0)=u_{1} \in W_{l} .
\end{aligned}
$$

Here $l \in \mathbb{N}$ is arbitrary but fixed.

We first note that by (2.2), $W_{l+1} \subset V$ and $W_{l} \subset W$ and thus from Theorem 2.1, there exists a unique global solution $u(t)$ to the problem (4.35)-(4.36) as constructed in Section 4.1.

Now we shall show that under the assumption of Theorem 2.2, the solution $u(t)$ to the problem (4.35)-(4.36) satisfies the properties (i), (ii), and (iii) in Theorem 2.2.

LEMMA 4.5. Let $l \in \mathbb{N}$. If $u(t) \in W_{l}$, then $u(t) \in W_{j}$ for $j=1, \ldots, l$. Moreover if $f \in L^{1}\left(0, \infty ; W_{l}\right)$, then $f \in L^{1}\left(0, \infty ; W_{j}\right)$ for $1 \leq j \leq l$. In fact, $f$ satisfies the following inequality

$$
\int_{0}^{\infty}\left|A^{j / 2} f(s)\right| d s \leq \int_{0}^{\infty}|f(s)| d s+\int_{0}^{\infty}\left|A^{l / 2} f(s)\right| d s \quad(1 \leq j \leq l) .
$$

PROOF. Let $\{E(\mu): 0 \leq \mu<\infty\}$ be the resolution of identity associated with $A$. Then we obtain by (2.1)

$$
\begin{aligned}
\left|A^{j / 2} u(t)\right|^{2} & =\int_{0}^{\infty} \mu^{j} d|E(\mu) u(t)|^{2} \\
& \leq \int_{0}^{1} d|E(\mu) u(t)|^{2}+\int_{1}^{\infty} \mu^{l} d|E(\mu) u(t)|^{2} \\
& \leq|u(t)|^{2}+\left|A^{l / 2} u(t)\right|^{2} \text { a.e. on }[0, \infty),
\end{aligned}
$$

which implies that $u(t) \in W_{j}, j=1, \ldots, l$ : Our second assertion immediately follows from (4.37) (see also [4]).

PROPOSITION 4.6. Let $u(t)$ be a unique global solution to the problem (4.35)(4.36). If in addition to the hypothesis of Theorem 2.1, $u_{0} \in W_{l+1}, u_{1} \in W_{l}$, and $f \in L^{1}\left(0, \infty ; W_{l}\right)$, then for $j=1,2, \ldots, l$,

$$
\begin{aligned}
& u(\cdot) \in L^{\infty}\left(0, \infty ; W_{j+1}\right) \cap B C\left([0, \infty) ; W_{j}\right), \\
& u^{\prime}(\cdot) \in L^{\infty}\left(0, \infty ; W_{j}\right) \cap B C\left([0, \infty) ; W_{j-1}\right) .
\end{aligned}
$$

In fact, we have on $[0, \infty)$,

$$
\begin{aligned}
& \left|A^{(j+1) / 2} u(t)\right| \leq \frac{C_{j}\left(\gamma_{0 j}\right)}{\sqrt{2 m_{0}}}, \\
& \left|A^{j / 2} u^{\prime}(t)\right| \leq C_{j}\left(\gamma_{0 j}\right),
\end{aligned}
$$


where

$$
C_{j}\left(\gamma_{0 j}\right)=\left(\sqrt{2}\left(\gamma_{0 j}^{2}\left(1+B_{0}+\delta+\delta^{2} / 2\right)+\delta B_{0} / \sqrt{m_{0}} \gamma_{0 j}\right)^{1 / 2}+2 \gamma_{0 j}\right)^{1 / 2} \quad\left(\gamma_{0 j}>0\right) .
$$

PROOF. We only prove (4.38) and (4.40). Statements (4.39) and (4.41) can be proved similarly. Let $u_{\lambda}(t)$ be a unique global solution to the problem (4.1) and (4.2).

Multiplying (4.1) by $2 A_{\lambda}^{j} u_{\lambda}^{\prime}(t)$ and $A_{\lambda}^{j} u_{\lambda}(t)$ and using Lemma 4.5 and a similar process as in the proof of Proposition 4.2, we obtain that for $j=1,2, \ldots, l$,

$$
\left|A_{\lambda}^{(j+1) / 2}(t)\right| \leq \frac{C_{j}\left(\gamma_{0 j}\right)}{\sqrt{2 m_{0}}} \text { on }[0, \infty) \text {. }
$$

Moreover, continuing in the same way as in the proof of Lemma 4.3 and using (4.42) we obtain $\left|A^{(j+1) / 2} u(t)\right| \leq C_{j}\left(\gamma_{0 j}\right) / \sqrt{2 m_{0}}$ and $u(\cdot) \in B C\left([0, \infty) ; W_{j}\right)$ for $j=1,2, \ldots, l$.

Finally, we shall show that $u^{\prime \prime} \in L^{\infty}\left(0, \infty ; W_{l-1}\right)$.

PROPOSITION 4.7. Let $u(t)$ be a unique global solution to the problem (4.35) and (4.36). If in addition to the hypothesis of Proposition 4.6,

$$
f \in L^{\infty}\left(0, \infty ; W_{l-1}\right)
$$

then $u^{\prime \prime}(\cdot) \in L^{\infty}\left(0, \infty ; W_{l-1}\right)$.

ProOF. Applying $A^{(l-1) / 2}$ to the both side of $(4.35)$, we have a.e. on $(0, \infty)$.

$$
A^{(l-1) / 2} u^{\prime \prime}(t)+M\left(\left|A^{1 / 2} u(t)\right|^{2}\right) A^{(l+1) / 2} u(t)+\delta A^{(l-1) / 2} u^{\prime}(t)=A^{(l-1) / 2} f(t) .
$$

So using (4.40) and (4.41) we have

$$
\left|A^{(l-1) / 2} u^{\prime \prime}(t)\right| \leq M_{5}(\delta)+\operatorname{ess} \sup \left\{\left|A^{(l-1) / 2} f(s)\right|: 0 \leq s<\infty\right\},
$$

where $M_{5}(\delta)=B_{2} C_{l}\left(\gamma_{0 l}\right) / \sqrt{2 m_{0}}+\delta C_{l-1}\left(\gamma_{0(l-1)}\right)$. Hence our assertion immediately follows from (4.43).

ProOF OF COROLlaRY 2.1. From (i) and (ii) of Theorem 2.2, we can easily check that

$$
u \in C\left([0, \infty) ; W_{l}\right) \cap C^{1}\left([0, \infty) ; W_{l-1}\right)
$$

Moreover, since $f \in C([0, \infty) ; H)$, we obtain from (4.35) and (4.44), $u^{\prime \prime}(\cdot) \in$ $C([0, \infty) ; H)$, which completes our proof. 


\section{Some applications}

Let $\Omega$ be a bounded domain in $\mathbb{R}^{n}$ with sufficiently smooth boundary $\partial \Omega$. We consider the initial boundary value problem with Dirichlet boundary conditions of the form

$$
\begin{gathered}
u^{\prime \prime}(x, t)+(-1)^{k} M\left(\int_{\Omega}\left|\nabla^{k} u(x, t)\right|^{2} d x\right) \Delta^{k} u(x, t)+\delta u^{\prime}(x, t) \\
=f(x, t), \quad x \in \Omega, t \in[0, \infty)
\end{gathered}
$$

$$
\begin{aligned}
& u(x, t)=\frac{\partial u}{\partial n}=\cdots=\frac{\partial^{k-1} u}{\partial^{k-1} n}=0, \quad x \in \partial \Omega, t \in[0, \infty) \\
& u(x, 0)=u_{0}(x), \quad u^{\prime}(x, 0)=u_{1}(x), \quad x \in \Omega
\end{aligned}
$$

where $\Delta$ and $\nabla$ are the Laplace operator and the gradient in $\mathbb{R}^{n}$, respectively, $\left|\nabla^{k} u\right|^{2}=$ $\left(\Delta^{k / 2} u\right)^{2}$ for even $k,\left|\nabla^{k} u\right|^{2}=\left|\nabla\left(\Delta^{(k-1) / 2} u\right)\right|^{2}$ for odd $k, M(\cdot)$ is a function satisfying (A.2) and $\delta>0$ is a constant and $n$ is the outer nomal to the boundary $\partial \Omega$.

Let $H=L^{2}(\Omega)$ be the Hilbert space with inner product (, ) and norm $\|\cdot\|$. Define a linear operator $A^{k}$ in $H$ by $A^{k} u=(-\Delta)^{k} u$ with domain $D\left(A^{k}\right)=H^{2 k}(\Omega) \cap H_{0}^{2 k-1}(\Omega)$. Here $H^{\gamma}(\Omega)$ is the usual Sobolev space of order $\gamma$ and $H_{0}^{\gamma}(\Omega)$ is the closure of $C_{0}^{\infty}$ in $H^{\gamma}(\Omega)$. Note that in this case we may regard the operator $A$ as $-\Delta$. It is well known that $A(=-\Delta)$ is a nonnegative selfadjoint operator with compact resolvent $(I+\lambda A)^{-1}$ for all $\lambda>0, D\left(A^{1 / 2}\right)=H_{0}^{1}(\Omega)$, and $\left\|A^{1 / 2} u\right\|=\|\nabla u\|, u \in D\left(A^{1 / 2}\right)$. By pointwise evaluation $u(x, t)=(u(t))(x)$, the problem (5.1)-(5.3) can be written in an abstract form (2.3)-(2.5).

Form Theorem 2.3 and Theorem 2.4, we obtain the following:

THEOREM 5.1. Let

$$
\begin{aligned}
\left(u_{0}, u_{1}, f\right) \in & \left(H^{2 k}(\Omega) \cap H_{0}^{2 k-1}(\Omega)\right) \times H_{0}^{2 k-1}(\Omega) \\
& \times\left(L^{1}\left(0, \infty ; H_{0}^{k}(\Omega)\right) \cap L^{\infty}(0, \infty ; H)\right) .
\end{aligned}
$$

Then there exists $\gamma_{0}>0$ satisfying the following. If

$$
\left\|u_{0}\right\| \leq \gamma_{0}, \quad\left\|\nabla^{2 k-1} u_{1}\right\| \leq \gamma_{0}, \quad \int_{0}^{\infty}\left\|\nabla^{2 k-1} f(\cdot, t)\right\| d t \leq \gamma_{0},
$$

then there exists a unique solution $u(t)$ on $[0, \infty)$ to the problem $(5.1)-(5.3)$ such that

$$
\begin{aligned}
& u \in L^{\infty}\left(0, \infty ; H^{2 k}(\Omega) \cap H_{0}^{2 k-1}(\Omega)\right) \cap B C\left([0, \infty) ; H_{0}^{2 k-1}(\Omega)\right), \\
& u^{\prime} \in L^{\infty}\left(0, \infty ; H_{0}^{2 k-1}(\Omega)\right) \cap B C\left([0, \infty) ; H^{2 k-2}\right), \quad u^{\prime \prime} \in L^{\infty}\left(0, \infty ; H^{2 k-2}\right) .
\end{aligned}
$$


Furthermore, if $u_{0} \in D\left(A^{k(l+1) / 2}\right), u_{1} \in D\left(A^{k l / 2}\right)$, and $f \in L^{1}\left(0, \infty ; D\left(A^{k l / 2}\right)\right) \cap$ $L^{\infty}\left(0, \infty ; D\left(A^{k(l-1) / 2}\right)\right)$ satisfies (5.4), then the solution $u(t)$ to the problem (5.1)-(5.3) satisfies

$$
\begin{aligned}
u & \in L^{\infty}\left(0, \infty ; D\left(A^{k(l+1) / 2}\right)\right) \cap B C\left([0, \infty) ; D\left(A^{k l / 2}\right)\right), \\
u^{\prime} & \in L^{\infty}\left(0, \infty ; D\left(A^{k l / 2}\right)\right) \cap B C\left([0, \infty) ; D\left(A^{k(l-1) / 2}\right)\right), \\
u^{\prime \prime} & \in L^{\infty}\left(0, \infty ; D\left(A^{k(l-1) / 2}\right)\right) .
\end{aligned}
$$

\section{Acknowledgment}

The author would like to thank the referee for helpful comments on the first version.

\section{References}

[1] E. H. Brito, 'The damped elastic stretched string equation generalized: existence, uniqueness, regularity and stability', Applicable Anal. 13 (1982), 219-233.

[2] R. W. Dickey, 'Infinite systems of nonlinear oscillation equations related to the string', Proc. Amer. Math. Soc. 23 (1969), 459-648.

[3] J. Dix and R. Torrejón, 'A quasilinear integrodifferential equation of hyperbolic type', Differential Integral Equations 6 (1993), 431-447.

[4] R. Ikehata and N. Okazawa, 'Yosida approximation and nonlinear hyperbolic equation', Nonlinear Anal. 15 (1990), 479-495.

[5] V. Lakshmikantham, S. Leela and A. A. Martynyuk, Stability analysis of nonlinear systems (Marcel Dekker, New York, 1989).

[6] G. P. Menzala, On classical solutions of a quasilinear hyperbolic equation, Memórias de Matemática da Universidade (Federal do Rio de Janeiro, 1978).

[7] R. Narasimha, 'Nonlinear vibration of an elastic string', J. Sound Vibration 8 (1968), 134-146.

[8] J. Y. Park and I. H. Jung, 'On a class of quasilinear hyperbolic equations and Yosida approximations', Indian J. Pure Appl. Math. 30 (1999), 1091-1106.

[9] J. Y. Park, I. H. Jung and Y. H. Kang, 'Some quasilinear hyperbolic equations and Yosida approximations', Bull. Korean Math. Soc. 38 (2001), 505-516.

[10] S. I. Pohožaev, 'On a class of quasilinear hyperbolic equations', Mat. Sb. 25 (1975), 145-158.

[11] H. Tanabe, Equations of evolution (Pitman, London, 1987).

[12] Y. Yamada, 'On some quasilinear wave equations with dissipative terms', Nagoya Math. J. 97 (1982), 17-39.

\section{Department of Mathematics}

Pusan National University

Pusan 609-735

Korea

e-mail: jyepark@pusan.ac.kr, ilhjung@pusan.ac.kr 